\title{
»NAVIgate Your SeT«. ZUR VIRTUOSITÄT VON DJS
}

\section{Lorenz Gilli}

»We all hit play« - mit diesen Worten beginnt Joel T. Zimmerman einen BlogEintrag im Jahr 2012. Zimmerman, besser bekannt als Deadmau5 (sprich: deadmaus), tritt mit diesem eine Diskussion um Live-Praktiken in der Electronic Dance Music (EDM) $)^{1}$ und die Relevanz des Beatmatching ${ }^{2}$ als technische Fertigkeit von $\mathrm{DJs}^{3}$ los (Deadmau5 2012, vgl. Attias 2013). Für den hier vorliegenden Beitrag habe ich dieses Statement als Startpunkt meiner Überlegungen genommen: Wenn DJs wirklich nur den Start-Knopf drücken, was sind dann die Fertigkeiten, die ein DJ haben muss und die dann so kunstfertig wie möglich - oder eben: virtuos - auszuführen sind? Damit meine ich nicht Fähigkeiten, die feiernden Massen verbal oder gestikulierend zu animieren, oder die Geschicklichkeit beim Selbstmanagement und bei der Selbstvermarktung oder die Kunstfertigkeiten als Studio-Produzent von EDM. Ich beziehe mich nur auf jene Aspekte, welche die musikalischklangliche Darbietung des DJing betreffen. Unter DJing verstehe ich das Ab-

1 »Electronic Dance Music « $(E D M)$ verwende ich, wie in der wissenschaftlichen Literatur üblich, als Überbegriff für eine Vielzahl von Genres wie House, Techno, Trance, Drum'n'Bass, Dubstep u.v.m. Im medialen Diskurs damit - oftmals abwertend - ein kommerziell ausgerichtetes Sub-Genre gemeint ist (vgl. Rietveld 2013a: 2), das ich (mit Holt 2017) als »EDM Pop« bezeichne.

2 Zum Begriff »Beatmatching « siehe Abschnitt 2. Fachbegriffe aus der Praxis und Kultur DJs (wie Beatmatching, Routine, Turntablism, Mixing, Programming etc.) verwende ich in der englischen Form, des Schriftbildes wegen aber werden sie bei Verwendung als Subjektiv groß geschrieben. Bei Verwendung in englischsprachigen Zitaten bleibt die Schreibweise wie im Original.

3 »DJ « leitet sich aus dem engl. Begriff »disk jockey« ab, ist aber mittlerweile fast nur mehr in der abbrevierten Form anzutreffen. Der Lesbarkeit halber wird der Begriff nur in seiner männlichen Form verwendet - leider entspricht eine männliche Dominanz in dieser Berufsgruppe auch empirisch den Tatsachen (vgl. Female Pressure 2013). Der Begriff »DJane « für weibliche DJs ist umstritten und wird daher von mir nicht verwendet - ggf. wird bei Relevanz der geschlechtlichen Identität des DJs der Begriff um »weiblich«bzw. »männlich« ergänzt. 
spielen und Mischen von medientechnisch fixierten Musik- und Klangstücken, die größtenteils von anderen Personen geschaffen wurden und die vom DJ vor Live-Publikum dargeboten werden. Der DJ ist hier also begrifflich strikt abzugrenzen von Akteuren, die v.a. ihre eigene Musik aufführen und die in der Literatur und Praxis als »Live-Act«, »Live-PA« oder »laptop performer« bezeichnet werden (vgl. Butler 2014: 6f.).

In den Texten, auf die im Folgenden referiert wird, fällt auf, dass einige der Autoren (Katz 2010; Veen/Attias 2011; Attias 2013; Reynolds 2013; Schüttpelz 2015) explizit die Begriffe »Virtuose « oder »Virtuosität « nutzen, jedoch eine Diskussion und Definition der Begriffe umgehen. Umgekehrt verweist Peter Wicke (2004) deutlich auf einige Kontinuitäten des Begriffs, nutzt inn aber für die populäre Musik auffallend vorsichtig. Dennoch weist er darauf hin, dass die Abwesenheit des Begriffs Virtuosität nicht auch die Abwesenheit der entsprechenden Fertigkeiten bedeute.

Dieser zurückhaltenden Anwendung des Begriffs in der populären Musik möchte ich mit diesem Beitrag bewusst entgegentreten und für eine Ausweitung des Konzepts auf die DJ-Kultur plädieren. Außerdem möchte ich dem von Hanns-Werner Heister (2004: 29/38) formulierten Desiderat der Ausdifferenzierung des Begriffs für unterschiedliche Musikkulturen, hier konkret: der DJ-Kultur, nachkommen. Als theoretische Ausgangslage für den Virtuositätsbegriff dient mir u.a. ebendieser Text »Zur Theorie der Virtuosität. Eine Skizze , in der Heister sieben Dimensionen von Virtuosität unterscheidet. Zusammenfassend könnte man musikalische Virtuosität bei ihm verstehen als jeweils sozial, historisch, technisch, kulturell und lokal bestimmte Höchstleistung von Spezialisten, die darin besteht, eine besondere und als solche erkennbare Schwierigkeit bei der Reproduktion eines musikalischen Werkes mit scheinbarer Mühelosigkeit und als materiell-technische Ausführung von zugrunde liegenden künstlerisch-geistigen Prozessen zu bewältigen. Anhand dieser begrifflichen Basis werde ich die Praktiken und Fertigkeiten des DJs untersuchen. Damit möchte ich zu einer Erweiterung oder Neuausrichtung des Konzepts der Virtuosität beitragen, die die Spezifika der DJ-Kultur und der Elektronischen (Tanz-)Musik mit berücksichtigt und somit einer Aktualisierung des Begriffs für zeitgenössische populäre Musik dient.

Der Beitrag gliedert sich in drei Abschnitte: Im ersten werde ich auf unterschiedliche kulturelle Ausformungen des DJs und insbesondere auf die damit verbundenen unterschiedlichen Inszenierungen des DJs eingehen. Hier werden insbesondere die Aspekte der kulturellen Bedingungen und der Sichtbarkeit bzw. Erkennbarkeit der Schwierigkeit angesprochen. Im zweiten Teil werde ich eine zentrale Fertigkeit des DJs, das Mixing, erläutern und im Besonderen auf den medialen Diskurs um die Mix-Technik des Beat- 
matching sowie dessen Relevanz für das DJing im digitalen Zeitalter eingehen. Hier steht grundlegend die Frage nach der Existenz und der Ausprägung der Schwierigkeit sowie ihrer scheinbar mühelosen bzw. risikoreichen Ausführung im Fokus. Im dritten und letzten Teil schließe ich eine theoretische Betrachtung an, inwiefern die aufgeworfenen Argumente praxistheoretisch und kulturanthropologisch zu belegen und $\mathrm{zu}$ rahmen sind. Diese Betrachtungen erweitern das Spektrum der Praktiken auf die zweite zentrale Fertigkeit des DJs, das Programming. Dieses umfasst v.a. die Auswahl der Tracks und den dramaturgischen Aufbau des gesamten DJ-Sets, womit die Dimension der materiell-technischen Ausführung von zugrunde liegenden künstlerisch-geistigen Prozessen in den Mittelpunkt rückt.

Zum Abschluss dieser Einleitung möchte ich mich selbst als Forscher im Feld positionieren und damit dem Leser meine eigene Herangehensweise an das untersuchte Phänomen aufzeigen. Dies scheint mir insbesondere aufgrund der Tatsache wichtig, dass ich das Phänomen nicht nur als kulturwissenschaftlich orientierter Medienwissenschaftler, sondern auch als Person mit Erfahrungen als DJ und Tänzer, vor allem in der Goa- und TechnoSzene, betrachte. Daher fließen meine eigenen Erfahrungen und Beobachtungen als DJ und Tänzer ebenso in die Thematik ein.

\section{Inszenierung des DJs}

Wenn ich Personen in meinem Umfeld erzählt habe, dass ich mich für einen Vortrag mit der Virtuosität von DJs beschäftige, dachten viele zuerst an die so genannten Turntablists und ihre beeindruckenden Mix-Techniken. ${ }^{4}$ Das ist kaum überraschend, da bei diesen Praktiken die bewusste Zurschaustellung der spieltechnischen Fertigkeiten prägnant hervortritt. Dieses Element identifizieren Heister (2004: 22ff.) und Wicke (2004: 235) als konstitutiv für Virtuosität.

Der Begriff »Turntablist« bzw. »Turntablism« wurde 1995 von DJ Babu geprägt und bezeichnet jene Art von DJing, bei der mit den Schallplatten und dem darauf gespeicherten Klang, in Verbindung mit der Reproduktionstechnologie des Plattenspielers, neue Klänge und neue Musik erschaffen wird: Turntablists »are what I would call performative DJs, who manipulate

4 Vgl. bspw. die Videos auf www.youtube.com/DMCworldchamps (Stand vom 3.1. 2017). 
recordings in live performance, transforming bits of preexisting recorded sounds into wholly new music « (Katz 2010: 125). ${ }^{5}$

Am anschaulichsten wird diese Neuschöpfung bei der Technik des Scratching: Scratches sind die rhythmischen Bewegungen des Plattentellers bei aufliegendem Tonarm und die dadurch produzierten, kratzigen Geräusche, die einerseits als rhythmische Erweiterung des zugrundeliegenden Grooves, andererseits als solistische Einzelleistung des DJs eingesetzt werden können (vgl. Rappe 2010, Tb. I: 161). Der dadurch entstehende Klang lässt u.U. den ursprünglichen Klang, aus dem der Scratch erwächst, nicht mehr erkennen. Neben dem Scratching sind die grundlegenden Techniken des Turntablism das Beatmatching (siehe Abschnitt 2) und das Breakbeating, bei dem einzelne rhythmische Passagen wiederholt, aneinandergereiht, geschichtet oder verschoben werden, sodass ein kontinuierlicher Rhythmus oder auch völlig neue rhythmische Pattern entstehen (vgl. ebd.; Rappe 2010, Tb. II: 133ff.). ${ }^{6}$

Turntablism stellt mit seiner bewussten Zurschaustellung artistischer Techniken eine Teilmenge des DJing in der HipHop-Kultur dar, die daneben auch das DJing im Club, im Radio und als Produzent umfasst. Die »DJBattle genannten Wettkämpfe sind eine Unterart des Turntablism, bei denen von einer Jury und/oder dem Publikum ein Sieger bestimmt wird:

»Once on stage, the battle DJ has a scant few minutes to demonstrate his (and occasionally her) virtuosity, originality, and crowd appeal. Battle routines are therefore rarely leisurely affairs, and aspire to demonstrate every facet of the turntablist's art, from boasting and dissing to scratching and beat juggling « (Katz 2010: 133).

Sowohl DJ-Battles im Speziellen als auch Turntablism-Veranstaltungen generell sind nicht als Tanzveranstaltung für ein Publikum intendiert:

»The tempos, timbres, textures and rhythms change every few seconds; what would clear a dance floor in record time (so to speak), instead draws the rapt attention and enthusiastic applause cheers of the crowd. (I can always tell newcomers to a turntablist showcase when they try, always unsuccessfully, to dance)«(Katz 2014).

DJ-Battles und Turntablism unterscheiden sich von anderen, als Tanzmusik konzipierten Veranstaltungsformaten in mehrfacher Hinsicht. Am auffälligs-

5 In diesem und allen folgenden Zitaten führe ich Hervorhebungen sowie Schreibund Tippfehler wie im Original an; der mitunter sehr häufig zu setzende Hinweis »[sic!] « entfällt so. Nur bei vom Original abweichender Darstellung weise ich explizit darauf hin.

6 Für eine detaillierte Erläuterung der Techniken des Mixing und der Klangmanipulation siehe Rappe (2010) oder Broughton/Brewsters (2002), spezifisch für das Scratching: Hansen (2010) oder Sonnenfeld (2011). 
ten ist die Länge der Performance: während die Performance eines BattleDJ nur wenige Minuten dauert, spannen sich DJ-Sets im HipHop und in der EDM über mehrere Stunden. Die Zusammenstellung der Tracks bzw. der Fragmente daraus und deren kunstfertige Kombination und Manipulation bei DJ-Battles wird als Routine bezeichnet, während im Club-Kontext vom Set oder DJ-Set gesprochen wird (vgl. Butler 2006: 202-254; 327). ${ }^{7}$ Diese Routines sind sehr stark geplant und einstudiert, wodurch kaum Raum für Improvisation bei der Performance bleibt. Allerdings wird im Vorfeld viel Zeit und Recherche darauf verwendet, die Routine auf das jeweilige Event und die einzelnen Gegner anzupassen.

Nach Katz (2010: 136) ist die Originalität der wichtigste der drei genannten Faktoren. Nach Heister ist Originalität jedoch als Bestandteil von Virtuosität zu denken. Für ihn ist Virtuosität gekennzeichnet durch den Doppelcharakter der technischen Perfektion: aus Üben und Wiederholen - fast bis zur Automatisierung - einerseits, und durch Ausprobieren und Experimentieren, durch »Veränderung des jeweils Neuen« andererseits (Heister 2004: 17).

Darüber hinaus geht es bei DJ-Battles darum, in der kurzen Zeit alle möglichen Facetten des Könnens zu zeigen - die bewusste Darstellung der Kunstfertigkeiten ist ein essentieller Bestandteil. Diese zu bewerten fällt jedoch oft schwer, da nur durch Zuhören die Routines oft nicht hinreichend verstanden und ihre Komplexität und ihr Anforderungsniveau nicht erkannt werden können. Die visuelle Information ist essentiell und wird von den DJs bewusst betont, teilweise durch sogenannte »body tricks« auch überbetont. Deren Einsatz als reines Show-Element ist umstritten und wurde seit den 1990er Jahren zugunsten einer höheren Musikalität wieder vermindert (vgl. Katz 2010: 135f.). Die Abgrenzung von Virtuosität als Meisterung einer echten technischen Schwierigkeit von einer effektvollen, aber bloß scheinbaren Schwierigkeit sieht Heister als besonders wichtig an und bezeichnet letzteres als »warenästhetische[n] Schein « und »Schatten« der Virtuosität (Heister 2004: 24). Battle-DJs sind sich also dieses Aspekts durchaus bewusst und reagieren sensibel auf Entwicklungen hin zu einem bloßen Schein von Virtuosität.

Die elaborierten Praktiken des Turntablism können aber auch als artistisches Element im Rahmen von längeren DJ-Sets oder Club-Nächten, also bei Tanzveranstaltungen für ein Publikum, eingesetzt werden. Bei derartigen Tanzveranstaltungen in Clubs oder bei Festivals ist die primäre Aufgabe des

7 Im HipHop-Kontext wird mit »DJ-Set« hingegen das Equipment des DJs bezeichnet, das in seiner Grundform aus zwei Turntables, einem Mischpult und Schallplatten als Klangquelle besteht (vgl. Rappe 2010, Tb. I: 222). 
DJs, den Anwesenden eine musikalische Basis zum Tanzen zu bieten. Dies gilt prinzipiell für alle Formen und (Sub-)Kulturen zeitgenössischer populärer Dance Music. In Bezug auf sozio-kulturelle und musikalisch-ästhetische Einbettung in eine bestimmte (Sub-)Kultur, auf die visuelle Inszenierung des DJs sowie die ökonomische Ausrichtung unterscheiden sich die diversen Veranstaltungen enorm, wie ich im Folgenden kurz aufzeigen werde.

Jan Kühn (2013: 167ff.) formuliert aus soziologischer Perspektive im Anschluss an Pierre Bourdieus »kulturelle Felder « und Sarah Thorntons »subkultureller Hierarchie « eine Opposition zwischen subkulturellen und massenkulturellen Szenen. Subkulturelle Szenen sind durch aktiv-produzierende Teilnahme der Szeneakteure und durch kleinwirtschaftlich strukturierte, oftmals nicht auf Profit, sondern Kostendeckung ausgerichtete Szenewirtschaft gekennzeichnet. Musik und Feierkultur stellen dabei den finalen Zweck dar. Die Größe der Veranstaltungen liegt typischerweise zwischen 150 und 2000 Menschen. Oftmals wird ihr »familiärer « Charakter betont, durch den über ähnliche Interessen und anschlussfähige soziale Hintergründe die Homogenität der Gruppe hergestellt wird. Lokalitäten, in denen solche Veranstaltungen ausgerichtet werden, bezeichnet Kühn als »Clubdisco (ebd.: 175). Aufgrund der verbreiteten Terminologie des »Club « und entsprechend des »Club DJ« (Fikentscher 2013: 125) bzw. »Club-DJ« (Wicke 2004: 237) verwende ich den Begriff »Club « in diesem, von Kühn formulierten Sinne. Kühns Feststellung, dass es sich nicht um herkömmliche Clubs mit verbrieften Mitgliedschaften und regelmäßigen Beiträgen handelt, sei hier explizit erwähnt (vgl. Kühn 2013: 175). Als typische Beispiele können die Berliner Clubs wie »Berghain« oder »about: blank« angeführt werden.

Den subkulturellen Szenen diametral gegenüber stehen bei Kühn die massenkulturellen Szenen, die »wesentlich nachfrage- und kapitalorientierter « (ebd.: 169) handeln. Die Akteure sind oftmals szenefern, richten sich an eine möglichst große Zielgruppe und zielen auf Profitgenerierung ab. Deswegen orientiert sich die musikalische Ausrichtung nicht an ästhetischen sondern kommerziellen Parametern (vgl. ebd.). Die konträren Veranstaltungsformen zum Club sind die Discotheken und Volksfeste: »Beliebige und undefinierte Menschenmassen, ob jung oder alt, tanzen zu populären Stücken der Musikindustrie aus Radio, Fernsehen und Charts, dem >Besten der 70er, 80er und 90er« (Kühn 2013: 172f.).

Ähnlich wie Kühn spricht auch Holt (2017) von Underground, und grenzt ihn gegen EDM Pop ab. Underground versteht er mit Bezug auf Richard Peterson (1997) als harten Kern der Genres (»hard core «) und EDM Pop als weiche Hülle (»soft shell«), bei der die Musik mit popmusikalischen Elementen angereichert wird, um sie einer größeren Anzahl von Menschen zugäng- 
lich und damit kommerziell erfolgreicher zu machen (vgl. Holt 2017: 4). Holt begreift EDM Pop, ähnlich wie Kühn, als »mass culture entertainment «, dessen ästhetische und inszenatorische Kernaspekte »theme park designs «, Feuerwerke und Bombast sind (ebd.: 3). EDM Pop-Festivals, die er untersucht und die als typische Beispiele gelten, sind »Tomorrowland« (Belgien, seit 2015 auch Brasilien) oder »Electric Daisy Carnival« und »Ultra Music« (beide USA). Hillegonda Rietveld bezeichnet solche Festivals und Inszenierungsstrategien mit Rückgriff auf Guy Debord als »Spektakel «, bei denen der DJ auf einer hohen Bühne platziert und in Licht gebadet wird. Der DJ bekommt nicht nur auditiv, sondern auch visuell eine hohe Aufmerksamkeit und wird als »god-like creative Frankenstein « (Rietveld 2013b: 80) gefeiert. Diese Art von Inszenierung des DJs wird als »Stadium-DJ«, »Arena-DJ« (ebd.) oder »Superstar DJ« (Hall/Zukic 2013: 106) bezeichnet.

Die hier aufgezeigten Oppositionen sind in der Praxis in vielerlei Mischformen zu finden. Daher sind diese als theoretisch gefasste Extrempole zu verstehen - die konkrete Ausgestaltung der einzelnen Veranstaltungen und insbesondere der Inszenierungen und der Praktiken des DJs, auf die ich in diesem Beitrag noch genauer eingehen werde, bewegen sich entlang einer Linie, die zwischen den beiden Extrempolen gedacht werden kann.

\subsection{Sichtbarkeit und Erkennbarkeit}

Durch die Positionierung des DJs auf einer spektakulär visuell inszenierten und hoch erhobenen Bühne wird nicht nur eine hohe Sichtbarkeit der Person gewährleistet, sondern auch eine strenge Hierarchie zwischen Publikum und DJ etabliert (vgl. Rietveld 2013b: 80). Auch wenn aufgrund der Größe der Veranstaltung die Sichtbarkeit nicht unbedingt mit Erkennbarkeit gleichzusetzen ist, in symbolischer Form ist sie jedenfalls vorhanden. In den so genannten »Underground«-Clubs hingegen kommt eine solche Hierarchie aufgrund der Ideale der Partizipation und Familiarität gar nicht oder nicht in diesem Maße vor. Im Gegenteil: In diesen gilt ein unsichtbarer oder zumindest nicht so stark im Vordergrund stehender DJ als Ideal (vgl. ebd.: 81ff.; Reynolds 2012).

Die Sichtbarkeit ist Grundbedingung für einen zentralen Bestandteil von Virtuosität, nämlich das »Ausstellen spieltechnischer Fertigkeiten und [der] zelebrierte[n] Veräußerlichung dieses Aspekts bis hin zum Sportlich-Zirzensischen « (Wicke 2004: 235). Heister betont zudem, dass die Beherrschung der Schwierigkeit auch als solche erkannt werden muss. Darüber hinaus stellt sich das Problem, dass die Virtuosität für den Laien und den Professionellen gleichermaßen als solche erkennbar sein soll, was Heister als »strukturelles 
Binnenproblem der Virtuosität « bezeichnet. Er unterscheidet zwischen einer exoterischen, also für alle erkennbaren, und einer esoterischen, also nur für Experten oder gar den Virtuosen selbst erkennbaren Virtuosität (Heister 2004: 22f.). Der Turntablist ist also eher ein exoterischer Virtuose: auch wenn nicht immer alle Ausführungen und Manipulationen verstanden und nachvollzogen werden können, so ist doch zumeist ein Gesamteindruck von Virtuosität auszumachen, dem mit den Strategien der »body tricks « - sofern maßvoll eingesetzt - nachgeholfen werden kann.

Beim Stadium- und beim Club-DJ ist die Sache anders gelagert: Während der Stadium-DJ zwar extrem sichtbar inszeniert ist, ist die Sichtbarkeit und die Nachvollziehbarkeit seiner instrumentellen oder technischen Ausführungen kaum gegeben. Beim Club-DJ wird das Problem noch einmal verschärft, wenn er nicht besonders sichtbar inszeniert wird; andererseits kann er aber aufgrund der kleineren Veranstaltungsgröße und des weniger erhöhten DJPults besser zu beobachten sein.

\section{Mixing - Beatmatching}

Kann man trotz geringer oder nicht vorhandener Sichtbarkeit dennoch von Virtuosität sprechen? Im Folgenden werde ich am Beispiel der Mix-Technik des Beatmatching zeigen, dass auch unsichtbare Praktiken als virtuos erkannt werden können. Dabei kommt ein bisher in der Literatur wenig beleuchteter Aspekt von Virtuosität zum Tragen: das Risiko. Darüber hinaus zeigt sich am Beispiel des Beatmatching außerdem, dass Virtuosität (bzw. ein Teilaspekt: die technische Beherrschung des Instruments) diskursiv als Machtinstrument eingesetzt und als Kriterium für Ein- und Ausschlüsse genutzt wird.

Das Mixing definiert Kai Fikentscher (2013: 125) als »the way a transition is accomplished from one record or track to another « und es ist neben dem Programming eine von zwei »principal skills involved in EDM DJing « (Butler 2006: 326). Fikentscher stellt damit die Gestaltung des Übergangs von einem Track zum nächsten innerhalb eines gesamten, ein- oder oft mehrstündigen DJ-Sets in den Vordergrund. Beim Turntablism hingegen steht, wie eingangs erwähnt, das kontinuierliche Hin- und Her-Blenden, das Loopen und Schichten von einzelnen Fragmenten aus mehreren Schallplatten im Vordergrund.

Eine Möglichkeit, diesen Übergang zwischen zwei Tracks zu gestalten, stellt das Beatmatching dar, das in Genres wie House, Techno oder Trance häufig eingesetzt wird. Dabei erfolgt das Überblenden zwischen zwei Tracks 
graduell, teilweise über mehrere Minuten hinweg. Auch die Manipulation der klanglichen Textur durch Filter und Equalizer erfolgt zumeist langsam, und abrupte Klangmanipulationen wie Scratchen oder ähnliches werden selten genutzt. ${ }^{8}$ Außerdem orientieren sich DJs beim Mix weniger an einzelnen Beats oder Takten, sondern an größeren Zyklen, was Butler (2006: 179ff.) als »multimeasure patterning « bezeichnet.

Rietveld bezeichnet diese Art des Mixings deshalb als »Slow-Mix« und identifiziert sie als Mix-Stil,

»der industriellen Techno, psychedelischen Trance und soulorientierten Garage verbindet, auch wenn sich diese Tanzmusikgenres in ihrem kulturellen Bezugsrahmen, ihrer >Textur`, in den bevorzugten Gegenrhythmen und im Tempo gravierend voneinander unterscheiden« (Rietveld 2001: 269).

Damit grenzt sie diesen Stil von den »abrupten Cutting-Techniken« bspw. im Reggae und im HipHop ab (vgl. ebd. u. 279f.). Simon Reynolds (2013: 576f.) argumentiert ähnlich und grenzt die beiden Stile mit »smooth« und »rough « voneinander $\mathrm{ab}$ und beschreibt ersteren auch als »long mix«, letzteren als »much shorter «.

Der Slow-Mix setzt voraus, dass die zu mixenden Tracks synchron in Bezug auf das Tempo als auch in Bezug auf den Beat abgespielt werden. ${ }^{9} \mathrm{Da}$ in vielen Fällen das Tempo der Tracks innerhalb eines Genres zwar ähnlich, aber nicht gleich ist, erfordert dies beim Auflegen mit Vinyl ein manuelles Anpassen des Tempos: das wird als Beatmatching oder als Beat Mixing bezeichnet (Butler 2006: 325, Rappe 2010, Tb. II: 133). DJ-Plattenspieler verfügen daher über einen Regler (»pitch control«), der diese Tempoänderung ermöglicht. Beim synchronen Abspielen über eine Zeitdauer, die wenige Sekunden übersteigt, müssen die Platten immer wieder leicht nachjustiert werden, was von den DJs meistens mit einem kurzen Anschieben oder Abbremsen einer der beiden Plattenteller ausgeführt wird. Diese Synchronizität über mehrere Minuten aufrechtzuerhalten und das Mixen beider Tracks zu einem neuen Klangstück ${ }^{10}$ durchzuführen, wird als »riding the mix« bezeichnet (Butler 2006: 93).

Beim DJing mit Vinyl beansprucht das Beatmatching beträchtliche Zeit und Aufmerksamkeit. DJs wie Richie Hawtin, Carl Cox oder Jeff Mills sind

8 Eine prominente Ausnahme stellt das oftmals abrupt durchgeführte Absenken und wieder Anheben der Bassfrequenzen durch den DJ dar (»withholding the beat«, Butler 2006: 91ff.).

9 Physikalisch gesprochen: synchron in Bezug auf Phase und Puls.

10 Butler bezeichnet das flüchtige Klangstück, das sich aus dem Mixen zweier Tracks ergibt und in dieser Form nicht medial fixiert existiert, als »third record« (vgl. Butler 2006: 94/243, 2014: 41ff.). 
bzw. waren für das meisterhafte »riding the mix« oder das Mixing mit drei oder vier Plattenspielern bekannt (vgl. Veen/Attias 2011; Attias 2013: 25; Reynolds 2013: 577). Das muss insofern historisiert werden, als dass z.B. Hawtin selbst seit vielen Jahren digitale DJ-Techniken nutzt und deren technologische Weiterentwicklung mit vorantreibt (vgl. McGlynn 2016). Cox legt auch nur noch selten mit Vinyl auf: Für das Abschluss-Set seiner 15-jährigen Residency im Club »Space« (Ibiza/Spanien) nutzte er zum ersten Mal nach zehn Jahren wieder Vinyl und betont den damit verbundenen nostalgischen Charakter (vgl. Turner 2016).

Die Fertigkeit des Beatmatching zu erlernen und fehlerfrei zu beherrschen ist keine triviale Angelegenheit, was viele DJs und Autoren betonen und ich selbst aus eigener Erfahrung weiß. Aktuelle DJ-Software wie "Serato DJ« oder »Traktor Pro« und digitale DJ-Controller führen die Anpassung von Tempo und Beat automatisch und unmittelbar aus. Zum Aufrufen dieser Funktion gibt es meist einen Knopf, der mit »Sync « beschriftet ist. Beatmatching als für DJs essentielle Fertigkeit ist also durch DJ-Software und den berühmt-berüchtigten »Sync-Button« eigentlich hinfällig geworden. Dennoch - oder gerade deswegen? - gibt es einen großen Diskurs um und widersprüchliche Sichtweisen über das Beatmatching als unumgängliche Fertigkeit, die ein DJ haben muss. Diesen Diskurs möchte ich im Folgenden kurz aufzeigen.

Einige Protagonisten lehnen Beatmatching als Kunstfertigkeit ab, wie der eingangs erwähnte Deadmau5: »>beatmatching < isnt even a fucking skill as far as i'm concered anyway. so what, you can count to 4. cool. i had that skill down when i was 3, so dont give me that argument please« (Deadmau5 2012). Allerdings ist Deadmau5 weniger daran gelegen, das automatisierte Beatmatching zu legitimieren. Ihm geht es laut eigener Aussage vor allem darum aufzuzeigen, dass EDM-Musiker auf der Bühne nicht »on the fly« (ebd.) neue, originale Tracks erschaffen. Vielmehr betont er, dass seine Skills dort glänzen, wo sie zu glänzen haben: im Studio und auf den Veröffentlichungen. Seine Live-Show hingegen sei sehr stark durchgeplant und mit den Licht- und Video-Elementen synchronisiert, sodass kaum Raum für Echtzeit-Aktivitäten bleibt: »[It] doesnt give me alot of >lookit me im jimi hendrix check out this solo stuff, because im constrained to work on a set timeline« (ebd.). Aus theoretischer Sicht entzieht sich Deadmau5 damit der Virtuositäts-Debatte, da der Virtuositätsbegriff fast immer an die Aufführung, nicht an die Komposition (oder in diesem Falle: Produktion) gekoppelt ist (vgl. Heister 2004: 27ff.; Wicke 2004: 235; Lösch 2004: 12). Allerdings findet im Diskurs eine Vermengung der Begriffe »DJ《 und »Laptop-Performer « statt - sowohl im Statement von Deadmau5 als auch in den Kommen- 
taren, wo scheinbar jeder, der EDM vor Live-Publikum aufführt, als DJ bezeichnet wird.

In ebendiesen Diskursen wird das Beatmatching immer wieder als Maßstab dafür herangezogen, ob sich jemand als DJ qualifiziert oder nicht, unabhängig davon, ob es sich um einen Laptop-Performer oder einen DJ handelt. Die kreative Fülle an Verunglimpfungen des Sync-Buttons bzw. der DJs, die sich auf ebenjenen beim Mixing verlassen, wird offenkundig, wenn man eine Suchmaschinen-Anfrage mit dem Begriff »Sync Button« oder »Sync Button Meme « durchführt. Diese Verunglimpfungen basieren auf dem Gedanken der meisterhaften Beherrschung des Instruments, einem Aspekt von Virtuosität, den neben Heister (2004: 17) auch Wicke (2004: 233) und Hans-Georg von Arburg (2006: 102) als zentral für Virtuosität ansehen.

Die Nutzung des Sync-Buttons zur automatischen Tempo-Synchronisation wird im Diskurs als illegitime Möglichkeit des Umgehens dieses technischen Aspektes, als >Schwindeln<, angesehen. Das Auflegen mit Turntables und Vinyl hingegen (so die implizite Argumentation) würde den Schwindler entlarven und somit die Spreu vom Weizen trennen, wie bspw. in diesem Internet-Mem des Blogs DJWORX ersichtlich ist.

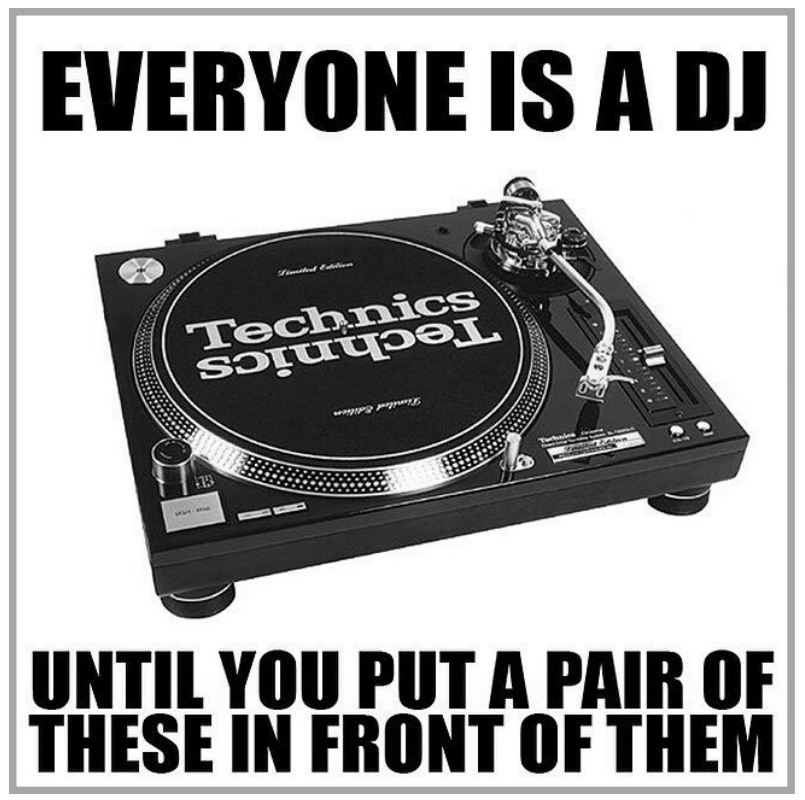

Abbildung 1: Internet-Mem (Settle 2015)

Hier dient also die virtuose Beherrschung des Instruments als Abgrenzungsund Ausschlussmerkmal gegenüber anderen, vermeintlich nicht-virtuosen DJ-Praktiken und gegenüber jenen Personen, die diese Praktik nicht zuverlässig beherrschen.

Andererseits wird aber durch das diskursive Aufrechterhalten des Beatmatching als vermeintlich notwendige Bedingung eine künstliche Virtuosität 
konstruiert, die aufgrund der Digitalisierung keine praktische Notwendigkeit mehr besitzt. Eine ähnliche Feststellung macht Wicke hinsichtlich der LivePerformance von Rockgitarristen:

»Lässt das musikalische Material [...] eine Demonstration der spieltechnischen Fertigkeiten nicht $\mathrm{zu}$, so werden erschwerende artistische Elemente in die Show eingebaut, die einen maximalen Körpereinsatz verlangen und allein dadurch selbst eine simple Spielfigur zu einer spieltechnischen Meisterleistung machen« (Wicke 2004: 237).

Als Beispiele führt Wicke unter anderem den Entengang von Chuck Berry oder das Gitarrenspiel von Jimi Hendrix mit Zähnen, Ellbogen und Zunge an. Auch die Body Tricks der Turntablists stellen teilweise eine solche bewusst eingesetzte Hürde dar (vgl. Katz 2010: 135). Allerdings betont Wicke (2004: 238), dass es sich dabei nicht um »Mätzchen« handelt, sondern dass diese artistischen Elemente Teil einer ritualisierten Performance-Strategie sind, die einen wichtigen Teil der gesamten Konzerterfahrung, des gesamten Rituals Rockkonzert, darstellt.

Analog kann man das Beatmatching und das Auflegen mit Vinyl als eine eigene Performance-Strategie der DJ-Kultur ansehen - auch wenn diese von vielen mittlerweile als nicht mehr zeitgemäß angesehen wird. Das Auflegen und Beatmatching mit drei oder vier Plattenspielern wäre dann noch eine Steigerung dieser Virtuosität. Außerdem wird bei Verwendung von Plattenspielern und Vinyl das Problem der Sichtbarkeit der manuellen Live-Praktiken reduziert, da hier die Praxis des DJs leichter sichtbar und nachvollziehbar ist als beim DJ mit Laptop, DJ-Software und Hardware-Controller. ${ }^{11}$ Insgesamt gesehen sind DJs nie so sichtbar und damit ihre Virtuosität nie so offen ausgestellt wie bspw. Rockgitarristen oder Geigenvirtuosen wie Nicoló Paganini. Turntables, die man sich ähnlich wie eine Gitarre umhängen kann (vgl. Claudius 2016), werden wohl kaum über den Status als Gag hinauskommen und sich nicht bei der breiten Masse durchsetzen. Welchen Stellenwert die Sichtbarkeit und die damit verbundenen virtuosen Praktiken in der EDM-Kultur, besonders in der Club-Kultur haben und dass auch unsichtbare Praktiken als Virtuosität gefasst werden können, werde ich im Folgenden aufzeigen.

11 Vgl. Butler (2014: 95ff.) zu diesem Problem, das er als »Conveying Liveness in Performance bezeichnet. 


\subsection{Risiko}

Die Automatisierung und Digitalisierung des DJing eliminiert nicht nur die mühsam erlernte Fertigkeit des Beatmatching, sondern darüber hinaus einen essentiellen Bestandteil des DJing, wie Tobias C. van Veen, Kulturwissenschaftler und Techno-DJ, aufzeigt:

»[The] >DJ culture< [...] loses touch not with the vinyl format, but with what constitutes the virtuosity of playing an inventive instrument - RISK. Without risk of fucking-up (trainwrecking, skipping the needle, a bad mix, etc.), there is no need for the human whatsoever «(Veen/Attias 2011). ${ }^{12}$

Die Nutzung einer Technologie, die bestimmte manuelle Fertigkeiten benötigt, ist mit einem Risiko verbunden und die Automatisierung dieser Fertigkeiten eliminiert auch das Risiko. Inwiefern aber das Eingehen eines solchen Risikos als Virtuosität verstanden werden kann, zeigt der Medientheoretiker Erhardt Schüttpelz (2015), der gerade das Handeln in einer potentiell misslingenden Situation als Grundlage von Virtuosität ansieht. Er beschreibt das Erlernen von Skills ${ }^{13}$, wie sie in technischen und wissenschaftlichen Laboren gefordert sind. Dabei bezieht er sich auf die Ausführungen von Hubert Dreyfus (2002) und gliedert den Erwerb von Fertigkeiten in drei Stufen: Der Novize erhält die Aufgabe in möglichst kleine und einfache Teilaufgaben zergliedert, die in einem geschützten Raum erlernt werden können. Nach und nach werden diese »Gebrauchsanweisungen « erweitert und zu »Richtlinien«. Der Fortgeschrittene hingegen verlässt den geschützten Raum und bekommt nur mehr grobe »Faustregeln« oder »Maximen« auf den Weg, die ihm als standardisierter Plan helfen, die Situation zu bewältigen. Durch eigene Fehler und Erfolgserlebnisse steigt die Kompetenz und er wird zu einem »technisch versierten, erfahrenen Techniker «. In einer unvorhergesehenen Situation »sieht er, was zu tun ist « und entscheidet aufgrund von Regeln und Analysen. Der Virtuose schließlich sieht zusätzlich, »wie das Ziel zu erreichen ist « und muss nicht auf Regeln und Analysen zurückgreifen, sondern kann sich auf die eigene, erlernte Improvisationsfähigkeit verlassen, um das Ziel zu erreichen (vgl. Schüttpelz 2015: 167-169). ${ }^{14}$

12 "Trainwrecking « bedeutet, dass der DJ durch falsche Trackauswahl oder unpassende Mix-Technik die Intensität der Musik abrupt abbremst - sprichwörtlich einen in voller Fahrt befindlichen Zug entgleisen lässt.

13 Der Begriff »Skill« bezeichnet im Englischen sowohl im alltäglichen als auch im wissenschaftlichen Sprachgebrauch »Geschicklichkeit«, »technisches Können« oder »Kunstfertigkeit « und hat insbesondere in der Praxistheorie in den letzten Jahren hohe Resonanz erfahren (vgl. Schüttpelz 2015: 153).

14 Schüttpelz expliziert nicht, weshalb er den Begriff »Virtuose« verwendet, zumal Dreyfus nur von »expert« als höchste (bei ihm fünfte) Stufe spricht. 
Hierin liegt für Schüttpelz der entscheidende Unterschied zwischen Fortgeschrittenen und Virtuosen:

»Der Virtuose in einem Gebiet, die wirkliche Expertin hingegen verlässt sich darauf, dass die Ressourcen der erfolgreichen Bewältigung einer technischen Aufgabe im Laufe ihrer Ausarbeitung noch auftauchen werden - eine Vorstellung, die in der geschützten Welt des Novizen Panik und in der Welt des Fortgeschrittenen den Wunsch nach fremden Ratschlägen und Hilfestellungen auslösen würde« (ebd.: 168-169).

Virtuosität ist also dann vorhanden, wenn trotz auftretender Schwierigkeiten und Gefahren die Aufgabe weiterhin ausgeführt und das Ziel konsequent verfolgt wird - ohne Hilfe von außen oder ohne Unterbrechung zur Planung der weiteren Schritte. Die Ausführung und die Planung der weiteren Handlungen erfolgen zeitgleich. Virtuosität ist demzufolge also ein der aktuellen Situation dienlicher Einsatz der Ressourcen und der eigenen Expertise - und damit auch ein oftmals unsichtbarer Prozess.

Veen hebt in seiner Beschreibung genau diese beiden Aspekte - Gleichzeitigkeit und Unsichtbarkeit - hervor:

»beatmatching in foreign set-ups to a full floor of hundreds/thousands and holding a mix for minutes on end is still a thrilling and dangerous experience, as correcting the record in-the-mix while EQing, slicing \& dicing and preparing the next record is a skill indeed. Doing so without the audience noticing corrections is quite an art« (Veen/ Attias 2011).

Das scheinbar mühelose Meistern einer schwierigen Aufgabe ist Bedingung für Virtuosität, mehr noch: es muss als Schwierigkeit auch erkennbar sein. Andererseits sollen aber »die technischen Probleme eben nicht als solche fühlbar werden und damit gar ablenken vom ästhetischen, sinnlich-geistigen Genuss « (Heister 2004: 26). Zwar müssen die technischen Schwierigkeiten irgendwie vermittelt werden, dürfen aber auch nicht zu sehr im Zentrum der Aufmerksamkeit stehen. Und ein bewusstes Heraustreten aus der laufenden Aufgabe zwecks Planung weiterer Schritte, wie es Schüttpelz für den Fortgeschrittenen beschreibt, würde einer Virtuosität sofort zuwider laufen.

Bemerkenswerterweise spricht Heister nicht von »Sichtbar-Werden« der Probleme, sondern vom »Fühlbar-Werden« und weicht damit terminologisch von einer rein visuell vermittelten Virtuosität ab. Dies ist besonders aufgrund der verminderten Sichtbarkeit des Club-DJ relevant. Denn ein meisterhaft durchgeführter Mix per Beatmatching kann auch rein auditiv erfahren werden:

»the overall mix is a much more driving experience for the dancefloor too. Though not all dancers might understand what is happening, I would argue 
that dancefloors instinctively respond to longer-held mixes as the affect of blended sounds creates heightened tension as the tracks play off each other. And once dancers realise you are mixing, bringing a record in and out repeatedly, and building the two records together to a mutual climax, the audience, well, err... comes along with you« (Veen/Attias 2011).

Hier stellt sich die Frage nach der Relevanz des Beatmatching im Vergleich zum Sync-Button. Natürlich spielt die Auswahl der Tracks eine große Rolle doch diese fällt nicht in das Regime des Beatmatching, sondern des Programming (siehe Abschnitt 3). In Bezug auf das Risiko beim Beatmatching stellt sich die Frage, wie der Rezipient am Dancefloor dieses Risiko erkennt: wohl nur durch kleine Imperfektionen, die sich im manuellen Beatmatching kaum vermeiden lassen (vgl. ebd.). Über diese wird das klangliche Ergebnis als Produkt einer hoch-riskanten manuellen Fertigkeit auditiv erfahrbar. Da aber nur erfahrene Hörer dies ohne weiteres erkennen können, kann man hier im Sinne Heisters (2004: 23) von einer esoterischen Virtuosität sprechen.

Virtuosität ist also offenbar nicht vereinbar mit Perfektion in der Ausführung, sondern eine erkennbare Gratwanderung. Der Sync-Button, der dieses Risiko eliminiert bzw. aus der Kontrolle des DJs und seinen manuellen Fertigkeiten hin zur verwendeten Hard- und Software verschiebt, eliminiert also auch die Virtuosität des Beatmatching per se.

Aus medienwissenschaftlicher Perspektive ist dieser Umstand interessant, da in diesen Momenten der hörbaren Überlagerung und Vermischung die zugrundeliegende Medientechnologie erkennbar und somit offengelegt wird. Malte Pelleter und Steffen Lepa (2007: 208) haben dies für das Sampling und das Scratching im HipHop aufgezeigt und es kann auf das Beatmatching übertragen werden.

Aber nicht nur eine medientechnologische Offenlegung findet hier statt, sondern auch eine medienästhetische. Yvonne Spielmann (2002: 245) konstatiert für die Filmästhetik, dass gerade in jenen Momenten, in denen die Schnitte, Brüche und »Kippfiguren« für den Rezipienten wahrnehmbar werden, (medien-)ästhetische Erfahrungen möglich werden. Analog erlaubt es auch das Beatmatching und dessen meisterhafte, aber doch erkennbare Ausführung der Überlagerung, eine auditive Erfahrung zu machen, dessen Reiz zusätzlich in der Flüchtigkeit und Einmaligkeit ihrer Ausführung besteht.

Aus der Beschreibung von Veen wird darüber hinaus ersichtlich, dass es sich beim Beatmatching nicht um eine handwerkliche Fertigkeit als bloßen Selbstzweck handelt, sondern um etwas, was im Dienste der Musik passiert: dass zwei Tracks jeweils ihre Stärken ausspielen (»play off each other «) und zu einem gemeinsamen Höhepunkt (»mutual climax«) hinarbeiten. Beat- 
matching ist hier lediglich die Technik, mit der diese performative KlangNeuschöpfung vom DJ ausgeführt wird. Die meisterhafte Ausführung muss aber einen irgendwie gearteten musikalischen Mehrwert bieten, um als Virtuosität zu gelten.

Dieser musikalische Mehrwert ergibt sich bei Heister aus einem Spannungsverhältnis zwischen der »materiell-technischen « und der »geistigen Dimension«. Den Endpunkt des Geistigen markieren dabei Kriterien wie »Ausdruck«, »Seele oder Sinn«, »Bewusstsein« oder »Darstellung der musikalischen Sache « (Heister 2004: 27f.). In populären Musikkulturen jedoch ist dieser Gegenpol weniger durch diese Aspekte, sondern durch die Bindung an den »ritualisierten Handlungskontext« (Wicke 2004: 243) gekennzeichnet, den der Künstler (in diesem Fall der DJ) gestalten und anführen muss. Die meisterhafte Beherrschung der Technik reicht dafür nicht aus:

»Gelingt es, die Welt der Apparate zu transzendieren, vermittels derselben, aber doch jenseits von ihnen den DJ und die tanzenden Körper untereinander und in Bezug aufeinander zu synchronisieren, dann erwächst eine Form von kollektivierter Selbsterfahrung daraus, die anderswo nicht zu haben ist« (ebd.: 242).

Wicke spricht in diesem Zusammenhang auch vom DJ als »Schamanen «. Heister begreift sogar den Schamanen des Spätpaläolithikums und seine Spezialisierung auf Ekstase- und Trance-Techniken als »früheste wenigstens in Umrissen greifbare Erscheinungsform der Virtuosität« (Heister 2004: 35). Die EDM-Kulturen schließen hier offenbar wieder an: nicht nur, dass ein Genre der EDM als »Trance« bezeichnet wird oder dass häufig Parallelen zwischen DJs und Schamanen bzw. EDM-Veranstaltungen und religiös-rituellen Zeremonien gezogen werden. ${ }^{15}$ Wicke schließt seinen Beitrag mit der Feststellung, dass Künstler der populären Kultur zwar nicht Virtuosen genannt werden, aber dass die »Ritualisierung durch Virtuosität [...] für die Popmusik viel zu fundamental [sei], um auch nur den Eindruck zuzulassen, es wären irgendwo Akteure am Werk, denen diese Fähigkeit abgeht « (Wicke 2004: 243).

15 Zum Beispiel: Der Techno- und Trance-DJ Sven Väth bezeichnet sich selbst als "Techno-Schamanen « (Schwarz 2002); der Goatrance-DJ Goa Gil ist selbst ein sadhu (hinduistischer »holy man«) und St John (2012: 91) bezeichnet inn als »dark yogi«; zum Thema insgesamt vgl. den Sammelband Rave Culture and Religion (St John 2004). 


\section{Programming - »Journey« und Navigation}

Die eben ausgeführten Punkte verdeutlichen, dass zu den Skills eines DJ auch die Auswahl der Tracks zählt: zum einen, da diese beim Mixing auch zueinander passen müssen und sich gegenseitig ergänzen können, und zum anderen, damit aus der mehrstündigen Aneinanderreihung von Tracks auch die beschriebenen ekstatischen Selbsterfahrungen möglich werden.

In der DJ-Kultur sind für die Gestaltung dieser Art von musikalischen Erfahrungen vor allem zwei Metaphern beliebt: »to take people on a journey« und »to tell a story (Reynolds 2013: 577; vgl. auch Broughton/Brewster 2002: 139; Fikentscher 2013: 130). Die konkrete Ausgestaltung dieser Geschichte oder Reise durch den DJ ist Bestandteil der zweiten zentralen Fertigkeit des DJing: dem Programming. Dieses beschreibt Fikentscher als "strategic combination of control over tempo, pacing, selection of repertoire and sound effects, including even the manipulation of dynamics and distribution (emphasis or de-emphasis) of frequency bands « (Reynolds 2013: 125). Der Begriff leitet sich also aus der Gestaltung des musikalischen Programms ab, und sollte daher nicht als starre Planung oder als Erstellen eines Algorithmus (auch nicht in einem metaphorischen Sinne) verstanden werden. Andere Autoren teilen die Fertigkeiten von DJs in drei Bereiche ein und führen neben dem Mixing und der Auswahl der Platten den »dramaturgischen Aufbau« (Reitsamer 2010: 170) oder »the psychological and spiritual aspects of the craft« (Reighley 2000: 147) an. Es ist unklar, ob Fikentscher diesen Bereich auch als dritten und eigenständigen Bereich fasst oder nicht: »The concept of a >musical journey< is closely related, not surprisingly, to that of a programme crafted by a DJ« (Fikentscher 2013: 130). Eine weitere Untersuchung und Ausdifferenzierung dieser Systematiken wäre noch notwendig - für diesen Beitrag fasse ich die genannten Aspekte unter das Programming und fokussiere auf die von Butler vorgeschlagene Zweiteilung in Mixing und Programming.

Auch Beatport, ein großer Online-Store für elektronische Tanzmusik, nutzt die Reise-Metapher gemeinsam mit der damit verwandten Metapher der Navigation, um das im Jahr 2016 eingeführte Tagging-Verfahren zu bewerben: Bei diesem Verfahren sind die Veröffentlichungen mit Tags, also Schlagworten, versehen, die dann als Such- und Filterkriterien sowohl beim Einkauf als auch in der eigenen Musikbibliothek für die Ordnung der Tracks genutzt werden können. Der User kann auch eigene Tags vergeben. Diese Tags beinhalten u.a. auch die vorgeschlagene Position des Tracks im Ablauf eines ganzen DJ-Sets (»Set Time«): Im entsprechenden Newsletter und auf 
der Website werden die drei Kategorien »Opening Tracks«, »Peak Hour Tracks« und »Closing Tracks« verwendet (vgl. Beatport 2016). In der Desktop-Anwendung von Beatport sind diese auf dreizehn verschiedene Tags weiter ausdifferenziert (vgl. Beatport 2017).

Ganz ähnliche Kategorien beschreibt der DJ und Musiker Solomun (Mladen Solomun) in einem Interview: »Ich habe jede Woche zwei Ordner mit vielleicht fünfzig, sechzig neuen Tracks, die ich noch mal unterteile, nach dem Schema Warm-up, Halb-Peaktime, Peaktime, geht richtig ab und morgens « (Solomun, zit. n. Klein 2014). Mit diesen und anderen Tags wie »mood «, »venue «, »bpm« oder »genre« kann der DJ seine Musikbibliothek ordnen und für den Auftritt vorbereiten. Allerdings zeichnet sich ein ClubAbend durch seine Offenheit und Unbestimmtheit aus, und es wird als essentiell angesehen, dass der DJ seine Musikauswahl an die lokalen und räumlichen Gegebenheiten, die Stimmung, das Publikum und die Auftrittszeit anpasst (vgl. Fikentscher 2000: 78-92; Montano 2009: 85-87). In den Diskursen um relevante Fertigkeiten des DJs findet sich diese Erwartung der Flexibilität und Anpassung häufig als Gegenargument zum Beatmatching als wichtigster Skill - so auch in den Kommentaren zum erwähnten Blog-Eintrag von Deadmau5: ein DJ definiere sich nicht so sehr dadurch, ob er das Beatmatching beherrsche, sondern durch »the journey s/he takes the dance floor on « (ultraaman 2012) oder dass er wisse, »how to work a crowd « (Horn 2014). Der Blog-Eintrag, aus dem das in Abb. 1 dargestellte Internet-Mem entnommen ist, wendet eben dieses Mem positiv und schließt mit der Abbildung einer enthusiastischen Menschenmenge und dem Schriftzug: »Everyone is a DJ if they have one of these in front of them « (Settle 2015).

\subsection{Social Navigation}

Um ein solches Handeln in einer offenen sozialen Situation besser beschreiben zu können, greife ich auf ein theoretisches Modell aus der KulturAnthropologie zurück: Social Navigation wird vom dänischen Kulturanthropologen Henrik Vigh in zwei Texten aus 2008 und 2009 als theoretisches Modell zur Beschreibung von Handeln in instabilen und bewegten sozialen Umwelten konzipiert. ${ }^{16}$ Die Betonung einer bewegten, fluiden Umwelt findet sich bereits im lateinischen Begriff »navigare «, der sich nur auf die Seefahrt und nicht auf die Bewegung an Land bezieht. Das Konzept selbst beschreibt Vigh wie folgt:

16 Vigh beschreibt in beiden Artikeln die instabile Situation im Bürgerkrieg gebeutelten Land Guinea-Bissau, betont aber die Anwendbarkeit des Modells auf unterschiedliche soziale Settings und Situationen (vgl. Vigh 2009: 420). 
»The concept [...] highlights motion within motion: it is the act of moving in an environment that is wavering and unsettled, and when used to illuminate social life it directs our attention to the fact that we move in social environments of actors and actants, individuals and institutions, that engage and move us as we move along (Vigh 2009: 420).

Social Navigation ist also die Art und Weise, wie Menschen sich in beweglichen Umwelten bewegen, und es betont die Unbestimmtheit von Subjekt und Umwelt. Damit unterscheidet sich das Konzept von anderen Theorien in den Sozialwissenschaften, die sich, laut Vigh, traditionellerweise entweder auf die Veränderung sozialer Formationen über die Zeit hinweg konzentrieren oder auf die Art und Weise, wie soziale Einheiten sich innerhalb sozialer Formationen bewegen. Social Navigation hingegen versucht, beides zeitgleich zu sehen und damit die Interaktivität der beiden zu beleuchten. Er würdigt zwar Bourdieus Praxis-Konzept, doch auch dieses geht für ihn in diesem Aspekt nicht weit genug (vgl. ebd.: 426f.).

Für dieses Handeln in bewegten Umwelten existiert in Guinea-Bissau ein eigener Begriff: »dubriagem«. Es ist ein Begriff der lokalen Verkehrssprache Krioulu, die auf dem Portugiesischen basiert. Vigh vergleicht es mit dem Englischen »muddling through « - auf Deutsch könnte man wohl »durchsteuern « oder »durchwursteln « dazu sagen. ${ }^{17}$ Dubriagem wird von Vigh beschrieben als »the ability to envision one's way through emergent and volatile socio-political cirumstances as well as being the actual practice of doing so « (ebd.: 424).

Besonders bedeutsam ist hier die Gleichzeitigkeit von Erkennen der Handlungsoptionen und der konkreten Ausführung dieser Optionen - trotz unklarer und jederzeit veränderlicher Rahmenbedingungen. Genau das hat Schüttpelz als entscheidenden Unterschied zwischen dem fortgeschrittenen Techniker und dem Virtuosen formuliert.

\subsection{Der DJ als »Social Navigator «}

Da auch EDM-Events häufig als bewegte und offene Umwelten gekennzeichnet sind, lässt sich dieses Konzept auf die Praxis des DJing übertragen und näher analysieren. Konkret fokussiere ich die Analyse auf die Zergliederung der Entscheidungsprozesse und ihrer Ausführungen.

17 Allerdings bezieht sich Vigh nicht auf das gleichnamige Konzept aus der Organisationssoziologie (»Muddling-Through «) nach Lindblom (1959), das die OnlineEnzyklopädie wikipedia.de mit »Sich-Durchwurschteln« übersetzt (https://de. wikipedia.org/wiki/Muddling-Through, Stand vom 29.1.2017). 
Aus Vighs Beschreibungen gehen einzelne Teilschritte hervor, in die sich die Social Navigation aufgliedern lässt. Bei der Suche nach Lösungen für die alläglichen Schwierigkeiten führt er drei Aspekte auf: »social life is surveyed, assumptions critically assessed and action tactile and tentative « (Vigh 2009: 422). Es handelt sich also um: Beobachtung der sozialen Umwelt, Bewertung der Annahmen und versuchsweise Handlung. Neben der Gleichzeitigkeit der Prozesse ist außerdem anzunehmen, dass diese Prozesse auch in zirkulären Schleifen ablaufen und sich stark gegenseitig bedingen. Dennoch bietet es sich an, sie für die theoretische Auseinandersetzung einzeln und getrennt zu betrachten.

Die Beobachtung, also die gezielte visuelle Wahrnehmung der sozialen Umwelt des DJs, kommt in der DJ-Kultur durch den Begriff »read the crowd « ${ }^{18}$ zum Ausdruck, der sich in vielen Diskursen und Aussagen wiederfindet. Beispielweise betont Fatboy Slim (Norman Cook):

»A good DJ is always looking at the crowd, seeing what they like, seeing whether it's working; communicating with them, smiling at them. A bad DJ is always looking down at the decks and just doing whatever they practised in their bedroom, regardless of whether the crowd are enjoying it or not « (Fatboy Slim, zit. n. Brewster/Broughton 2006: 20).

Allerdings ist diese Beobachtung nicht nur eine monodirektionale Aktivität, die der DJ von seiner (mal mehr, mal weniger) erhöhten Kanzel her ausführt, sondern ein bidirektionales Verhältnis, eine Kommunikationsbeziehung (vgl. Rietveld 2013b: 80ff.). Auch sollte hervorgehoben werden, dass die visuelle Wahrnehmung nicht die einzige Modalität ist, in der diese Kommunikationsbeziehung vonstattengehen kann. Ein verbreiteter Begriff ist jener des »vibe«, der vom DJ >gefühlt < werden muss (»feel the vibe «, Fikentscher 2000: 91). DJ Calemma (Giovanni Calemma) hingegen spricht von »empathic communication« (Calemma 2015). Obwohl diese Begrifflichkeiten in den Aussagen oftmals nicht einer theoretischen Fundierung genügen, zeigen sie in ihrer alltäglichen Verwendung doch auf, dass zumindest subjektiv nicht nur die visuelle Wahrnehmung als Sinnesmodalität zur Kommunikation eingesetzt wird.

Der DJ ist aber nicht nur Kommunikationspartner, er ist auch Teilnehmer, was sich maßgeblich auf die Art und Weise auswirkt, wie die Bewertung der Annahmen durchgeführt werden kann. Für David Mancuso, einer der Gründungsfiguren der Discokultur, ist der DJ zugleich Performer und Rezipient und hat »one foot on the dancefloor and one in the booth « (zit. n. Brewster/Broughton 2006: 20). Butler hat diese Doppelfunktion als Perfor-

18 Auf Deutsch etwa: »das Publikum lesen«. 
mer und Rezipient weiter ausgeführt und mit seinem Konzept der »listener orientation « beschrieben, wie Laptop-Performer und DJs die Möglichkeit haben, der Musik zuzuhören, während diese ohne ihr Zutun weiterspielt. Das unterscheidet sie von Musikern, die auf traditionellen Instrumenten ${ }^{19}$ spielen: »DJs are continuously evaluating the current configuration of sounds; determining if, when, and how it should change; and thinking about what sort of sound or record should follow next«(Butler 2014: 106). DJs bewerten also die momentan gespielte Musik aus der Perspektive des Hörers heraus und treffen gleichzeitig Entscheidungen darüber, welche weiteren Schritte zu setzen seien, also: welche Annahme(n) als passend zu bewerten sei(en).

Die Umsetzung dieser Annahme(n) erfolgt als versuchsweise Handlung: So empfehlen die beiden Autoren Frank Broughton und Bill Brewster für die Entscheidung hinsichtlich des nächsten Tracks, dass zu jeder potentiellen Auswahl weitere fünf Anschluss-Tracks vorhanden sein sollen, um je nach Reaktion des Publikums in die eine oder andere Richtung weiter zu gehen. »In your mind you should see a network of records and their possible connections. « (Broughton/Brewster 2002: 138). Das bedeutet, dass jeder Track auch immer ein Test ist, wie das Publikum reagiert und was anschließend zu tun sei. Dieses Vorgehen wurde mir von DJ Calemma in einem Interview bestätigt (vgl. Calemma 2015). Der DJ muss also die Bewegtheit und Offenheit der sozialen Umwelt in eine Offenheit der Track-Auswahl, des Programming übersetzen. Insofern könnte man hier von einer Erweiterung der Navigation in das Feld des Klanglich-Musikalischen und somit des Ästhetischen, also von einer »aesthetic navigation«, sprechen.

Die bereits mehrfach betonte Gleichzeitigkeit von Planung und Handlung bestimmt die Qualität des musikalischen Outputs, insbesondere das Timing rhythmischer Aktivitäten wie die des Beatmatching. Dies stellt auch Rosa Reitsamer in ihrer soziologischen Untersuchung von DJs fest:

»Im Zusammenspiel aus dem Hören des anberaumten Musikstücks über Kopfhörer, dem Hören des gerade gespielten Musikstücks über die Lautsprecher im Club und den Bewegungsabläufen der Hände bei der Bedienung der technischen Geräte verringert jedes Überlegen der Arbeitsschritte und ihrer Abfolge die Wahrscheinlichkeit einer präzisen Übereinstimmung der unterschiedlichen Rhythmen und Takte der Musikstücke« (Reitsamer 2010: 170, Hervorhebungen LG).

Dieses »Technik-Wissen«, wie Reitsamer den soeben beschriebenen Wissensbestand der DJs bezeichnet, ist verkörpertes Wissen, auf das DJs intuitiv

19 Der Begriff »traditionelle Instrumente « wird von Butler (2014: 105) in Abgrenzung zum Instrumentarium der elektronischen Musik genutzt. 
zugreifen können - ein bewusstes Überlegen der auszuführenden Schritte würde das Ergebnis verschlechtern und damit eine möglicherweise virtuose Performance verhindern. Aber nicht nur das Technik-Wissen, sondern auch das »Szene-Wissen « wird über körperliche Lernprozesse akkumuliert und wieder intuitiv abgerufen. Unter Szene-Wissen versteht sie unter anderem das Wissen, die Stimmung des Publikums durch entsprechende Wahl der Art des Mixings und der auszuwählenden Tracks positiv zu lenken. Die Auswahl der Tracks bildet den letzten der drei Wissensbestände von DJs, das »MusikWissen« (vgl. ebd.: 167ff.).

Der bereits genannte Vibe dient in der DJ-Kultur zur Beschreibung der Stimmung des Publikums, auf die der DJ entsprechend den Bedürfnissen der Crowd reagieren soll - andere Begriffe sind Energy oder Atmosphere. Umgekehrt ist der Vibe wiederum stark durch den DJ bestimmt, wodurch eine Rückkoppelung bzw. Feedbackschleife entsteht (vgl. Fikentscher 2000: 79ff.).

Damit ist die Arbeit des Club-DJ als ein performativer Akt im Sinne Erika Fischer-Lichtes beschrieben: Für sie ist es konstitutiv für performative Akte, dass keine klare Trennung zwischen Akteur und Publikum, also keine Subjekt-Objekt-Dichotomie besteht, sondern dass das Verhältnis der Anwesenden als »leibliche Ko-Präsenz « besteht und verstanden wird. Die Anwesenden stehen durch eine Feedback-Schleife in ständigem, wechselseitigem Austausch, wodurch Ereignisse entstehen, die nicht völlig planbar und vorhersagbar sind (vgl. Fischer-Lichte 2004: 58ff.).

Da die Auswahl der Tracks nicht von vorherein feststeht, sondern oftmals stark improvisierend vorgenommen wird, muss sie auch als Teil der Performance und somit als mögliches Feld, in dem Virtuosität sich manifestieren kann, betrachtet werden. Eine virtuose Selection wäre also eine Track-Auswahl, bei der der DJ den momentan existierenden Vibe richtig aufzunehmen weiß, einen Track auswählt, der diese Stimmung in die richtige Richtung weiterlenken kann und der vom DJ mit der passenden MixTechnik in den gerade gespielten Track eingeblendet wird. Diese Mix-Technik und der eingespielte Track würden dabei in positiver Weise Spannung aufbauen, die dann im richtigen Moment eingelöst würde und somit die Euphorie und Ekstase beim Publikum verstärken würde.

Der Rahmen dafür, also welche Tracks, Stile und (Sub-)Genres in Frage kommen und dem DJ zur Auswahl stehen, ist dabei weder völlig offen noch willkürlich eingeschränkt, sondern unterliegt szene-internen Regulationsprozessen und Diskursen (vgl. Bonz 2014: 243ff.; Doehring 2015: 148ff.). Vor allem aber ist es ein höchstens in Umrissen kanonisiertes und nicht durch formale musikalische Bildung erlernbares Wissen, sondern hauptsächlich 
durch aktive Teilnahme an der entsprechenden (Sub-)Kultur erlernbar (vgl. Reitsamer 2010: 167ff.; Bonz 2014: 243ff).

Es handelt sich also um einen Wissensbestand, den DJs beherrschen müssen, ähnlich den musik-immanenten Einschränkungen und Grenzen, die der Virtuose zu beachten hat und die das ihm zur Verfügung stehende musikalische Material begrenzen. Heister (2004: 29) nennt dies »das Externe«. Dieses Externe ist bei der DJ-Kultur und ihren individuellen, lokal geprägten und schnell wandelbaren Szenen diffus, fragmentiert und ständig im Wandel - und gerade deshalb ist das meisterhaft ausgeführte und situativ angepasste Schöpfen aus diesem musikalischen Fundus eine virtuose Angelegenheit. Oder wie es Brewster und Broughton (2006: 16) formulieren: »A DJ's job is to channel the vast ocean of recorded sound into a single unforgettable evening. «

\section{Fazit}

Die unterschiedlichen kulturellen Ausformungen und Inszenierungen des DJs bestimmen die Sichtbarkeit und Erkennbarkeit. Damit sind die Möglichkeiten der bewussten Zurschaustellung der eigenen Kunstfertigkeiten als eine Dimension von Virtuosität betroffen. Im ersten Abschnitt habe ich gezeigt, dass Turntablists und Battle-DJs diese Dimension offensiv nutzen und somit als exoterische Virtuosen angesehen werden können. Stadium-DJs und ClubDJs hingegen sind mit dem Problem der geringeren Sicht- bzw. Erkennbarkeit konfrontiert.

Im zweiten Abschnitt habe ich die Mix-Technik des Beatmatching für die sog. »Slow-Mix«-Stile analysiert. Diese Technik ist beim besonders langen Halten des Mixes (»riding the mix«) oder bei der Ausführung mit drei oder vier Plattenspielern besonders schwierig und wird bereits als virtuos bezeichnet. Die damit adressierte Dimension der meisterhaften Beherrschung des Instruments droht mit dem Sync-Button hinfällig zu werden; sie ist aber auch jene Dimension, die im Diskurs um das Beatmatching besonders betont und diskursiv als Machtinstrument eingesetzt wird. Darüber hinaus kann das Beatmatching als eine künstliche Virtuosität im Rahmen einer ritualisierten Performance-Strategie genutzt werden.

Das Risiko, das mit dem Beatmatching in Live-Situationen verbunden ist und sich darin manifestiert, dass Unvorhergesehenes gemeistert und gleichzeitig die Aufgabe weiterhin erfüllt wird, ist bei Schüttpelz das Kriterium, das den Virtuosen vom Techniker unterscheidet. Und da dieses Risiko über das Beatmatching vom Rezipienten rein auditiv erkennbar wird, kann es als 
esoterische Virtuosität bezeichnet werden. Der musikalische Mehrwert, der diese Praxis von einer rein materiell-technischen Ausführung unterscheidet, ergibt sich durch den vom DJ hergestellten rituellen Rahmen, in dem die Rezipienten eine einzigartige Selbsterfahrung erleben.

Der dritte Abschnitt hat den zweiten wichtigen Skill von DJs, das Programming, beleuchtet, die mit Navigation nicht nur als Metapher, sondern auch als theoretisches Modell umrissen werden kann. Fluide Situationen erfordern vom DJ ein gleichzeitiges Planen und Ausführen der drei Teilaspekte Beobachtung, Bewertung der Annahmen und versuchsweise Handlung. Alle drei Teilaspekte werden in der Praxis in einzigartiger, für die DJ-Kultur spezifische Art und Weise ausgeführt und formen letztendlich die Stimmung des Publikums, den Vibe.

Es ist vor allem die sich entfaltende, auditiv wie leiblich erfahrbare Klangwirkung, die am Ende über die Virtuosität des DJs entscheidet. Virtuosität beim DJ geht über eine spieltechnische Meisterschaft hinaus und muss um eine soziale sowie ästhetische Komponente erweitert werden; dementsprechend müssen DJs als virtuose Agenten in einer unklaren, veränderlichen Umwelt angesehen werden, in der Technologie, Klänge und Menschen vernetzt und wechselseitig miteinander in dauerndem Austausch stehen.

\section{Literatur}

Arburg, Hans-Georg von (2006). »(An)Gewandte Künste. Virtuosität als Problem der Ästhetik im technischen Zeitalter zwischen Idealismus und Historismus. " In: Virtuosität Kult und Krise der Artistik in Literatur und Kunst der Moderne. Hg. v. dems. Göttingen: Wallstein, S. 102-126.

Attias, Bernardo Alexander (2013). "Subjectivity in the Groove: Phonography, Digitality and Fidelity."In: DJ Culture in the Mix: Power, Technology, and Social Change in Electronic Dance Music. Hg. v. Bernardo Alexander Attias, Anna Gavanas und Hillegonda Rietveld. London: Bloomsbury, S. 15-50.

Beatport (2016). Navigate Your Set. E-Mail-Newsletter (Stand vom 27.5.2016), https: / /www.beatport.com/navigate-your-set (Stand vom 31.3.2017).

Beatport (2017). 1 Million Tagged Tracks At Your Fingertips. A Sleeker Way to Browse and Build Sets, https://www.beatport.com/1milliontags (Stand vom 29.1.2017).

Bonz, Jochen (2014). »Acid House als Grenze des praxeologischen Kulturverständnisses. Zum Realismus der sensuellen Ethnographie.« In: Ethnographien der Sinne. Wahrnehmung und Methode in empirisch-kulturwissenschaftlichen Forschungen. Hg. v. Lydia Maria Arantes und Elisa Rieger. Bielefeld: transcript, S. 233-250.

Brewster, Bill / Broughton, Frank (2006). Last Night a DJ Saved my Life. The History of the Disc Jockey. London: Headline.

Broughton, Frank / Brewster, Bill (2002). How to DJ (Properly). The Art and Science of Playing Records. London: Bantam. 
Butler, Mark J. (2006). Unlocking the Groove. Rhythm, Meter, and Musical Design in Electronic Dance Music. Bloomington, IN: Indiana University Press.

Butler, Mark J. (2014). Playing with Something that Runs. Technology, Improvisation, and Composition in DJ and Laptop Performance. New York: Oxford University Press.

Calemma, Giovanni (2015). Persönliches Interview am 13. August in Belluno: Sonica Dance Festival (Gedächtnisprotokoll).

Claudius (2016). »Vom DJ zum Rockstar? Scratchocaster - Turntable zum Umhängen.« In: Gearnews vom 29. Februar, https://www.gearnews.de/vom-dj-zumrockstar-scratchocaster-turntable-zum-umhaengen (Stand vom 29.1.2017).

Deadmau5 (2012). »All DJs Are Glorified Button Pushers.«In: Gizmodo vom 22. Juni, http://gizmodo.com/5921053/all-djs-are-glorified-button-pushers?comme $\mathrm{nt}=50924491$ \#comments [republished from http://deadmau5.tumblr.com/post/ 25690507284/we-all-hit-play] (Stand vom 15.11.2016).

Doehring, André (2015). »Andrés's > New For U<: New for Us. On Analysing Electronic Dance Music. «In: Song Interpretation in 21st-Century Pop Music. Hg. v. Ralf von Appen, André Doehring und Allan F. Moore. Farnham: Ashgate, S. 133-155.

Dreyfus, Hubert L. (2002). »Intelligence without Representation. Merleau-Ponty's Critique of Mental Representation. « In: Phenomenology and the Cognitive Sciences 1, S. 367-383.

Female Pressure (2013). »Press Statement - 8 March 2013 - International Women's Day.« In: Female:Pressure vom 8. März, http://www.femalepressure.net/ pressrelease.html\#english (Stand vom 31.1.2017).

Fikentscher, Kai (2000). You Better Work! Underground Dance Music in New York City. Hanover, NH: University Press of New England.

Fikentscher, Kai (2013). " It's not the Mix, it's the Selection<. Music Programming in Contemporary DJ Culture.«In: DJ Culture in the Mix: Power, Technology, and Social Change in Electronic Dance Music. Hg. v. Bernardo Alexander Attias, Anna Gavanas und Hillegonda Rietveld. London: Bloomsbury, S. 123-150.

Fischer-Lichte, Erika (2004). Ästhetik des Performativen. Frankfurt/M.: Suhrkamp.

Hall, Mirko J. I Zukic, Naida (2013). »The DJ as Electronic De-Territorializer.« In: DJ Culture in the Mix: Power, Technology, and Social Change in Electronic Dance Music. Hg. v. Bernardo Alexander Attias, Anna Gavanas und Hillegonda Rietveld. London: Bloomsbury, S. 103-122.

Hansen, Kjetil Falkenberg (2010). The Acoustics and Performance of DJ Scratching. Analysis and Modeling. Stockholm: KTH School of Computer Science and Communication.

Heister, Hanns-Werner (2004). »Zur Theorie der Virtuosität. Eine Skizze. « In: Musikalische Virtuosität. Hg. v. Heinz von Loesch, Ulrich Mahlert und Peter Rummenhöller. Mainz u.a.: Schott, S. 17-38.

Holt, Fabian (2017, in Print). „EDM Pop. A Soft Shell Formation in a New Festival Economy. «In: Weekend Societies. Electronic Dance Music Festivals and EventCultures. Hg. v. Graham St John. London: Bloomsbury. Online: https://ruc-dk. academia.edu (Stand vom 13.1.2017)

Horn, Leslie (2012). [Online-Kommentar zu Deadmau5 (2012)]. In: Gizmodo vom 25. Juni, http://gizmodo.com/i-dont-disagree-with-this-but-djs-good-ones-knowhow-t-1578513130 (Stand vom 29.1.2017)

Katz, Mark (2010). Capturing Sound. How Technology Has Changed Music. Berkeley, CA: University of California Press.

Katz, Mark (2014). »Hip-Hop Turntablism, Creativity and Collaboration. Sophy Smith. Farnham: Ashgate, 2013« [Rezension]. In: Dancecult. Journal for Electro- 
nic Dance Music Culture 6, Nr. 1, https://dj.dancecult.net/index.php/dance cult/article/view/487/468 (Stand vom 16.2.2017).

Klein, Holger (2014). »MOVE D \& SOLOMUN Über das Auflegen.«In: Groove vom 26. Dezember, http://groove.de/2014/12/26/move-d-solomun-ueber-das-auflegen (Stand vom 29.1.2017).

Kühn, Jan-Michael (2013). ») Underground und Kulturproduktion. Die Rolle von Distinktionen beim Veranstalten Berliner Techno-Partys. «In: Akustisches Kapital: Wertschöpfung in der Musikwirtschaft. Hg. v. Bastian Lange, Hans-Joachim Bürkner und Elke Schüßler. Bielefeld: transcript, S. 161-184.

Lindblom, Charles E. (1959). »The Science of Muddling-Through.«In: Public Administration Review 19, S. 79-88.

Loesch, Heinz von (2004). »Virtuosität als Gegenstand der Musikwissenschaft. « In: Musikalische Virtuosität. Hg. v. Heinz von Loesch, Ulrich Mahlert und Peter Rummenhöller. Mainz u.a.: Schott, S. 11-16.

McGlynn, Declan (2016). »Cover Feature: DJ Mag Meets Richie Hawtin. We Meet Futurist Techno Maestro Richie Hawtin for our ADE Issue...« In: DJ Mag vom 14. Oktober, https://djmag.com/news/cover-feature-dj-mag-meets-richie-hawtin (Stand vom 29.1.2017).

Montano, Ed (2009). „DJ Culture in the Commercial Sydney Dance Music Scene. «In: Dancecult. Journal for Electronic Dance Music Culture 1, Nr. 1, S. 81-93; https://dj.dancecult.net/index.php/dancecult/article/view/272 (Stand vom 16.2.2017).

Pelleter, Malte / Lepa, Steffen (2007). »Sampling als kulturelle Praxis des HipHop. «In: HipHop meets Academia. Globale Spuren eines lokalen Kulturphänomens. Hg. v. Katrin Bock, Stefan Meier und Gunter Süss. Bielefeld: transcript, S. $199-213$.

Peterson, Richard A. (1997). Creating Country Music. Fabricating Authenticity. Chicago, IL: University of Chicago Press.

Rappe, Michael (2010). Under Construction. Kontextbezogene Analyse afroamerikanischer Popmusik. 2 Teilbände. Köln: Verlag Döhr.

Reighley, Kurt B. (2000). Looking for the Perfect Beat. The Art and Culture of the DJ. New York u.a.: Pocket Books.

Reitsamer, Rosa (2010). »Wissen im Plural. Was wissen DJs?« In: Körper, Wissen, Geschlecht. Geschlechterwissen und soziale Praxis II. Hg. v. Angelika Wetterer. Sulzbach/Taunus: Helmer, S.165-182.

Reynolds, Simon (2012). »How rave music conquered America. In: The Guardian vom 2. August, https://www.theguardian.com/music/2012/aug/02/how-ravemusic-conquered-america (Stand vom 29.1.2017).

Reynolds, Simon (2013). Energy Flash. A Journey through Rave Music and Dance Culture. London: Faber and Faber (New and revised ed.).

Rietveld, Hillegonda (2001). »Im Strom des Techno. -Slow-Mix<-DJ-Stile in der Dance Music der 90er Jahre.«In: Rock- und Popmusik. Hg. v. Peter Wicke (= Handbuch der Musik im 20. Jahrhundert 8). Laaber: Laaber, S. 267-311.

Rietveld, Hillegonda (2013a). »Introduction.«In: DJ Culture in the Mix: Power, Technology, and Social Change in Electronic Dance Music. Hg. v. Bernardo Alexander Attias, Anna Gavanas und Hillegonda Rietveld. London: Bloomsbury, S. 1-14.

Rietveld, Hillegonda (2013b). "Journey to the Light? Immersion, Spectacle and Mediation. "In: DJ Culture in the Mix: Power, Technology, and Social Change in Electronic Dance Music. Hg. v. Bernardo Alexander Attias, Anna Gavanas und Hillegonda Rietveld. London: Bloomsbury, S. 79-102. 
Schüttpelz, Erhard (2015). "Skill, Deixis, Medien.«In: Mediale Anthropologie. Hg. v. Christiane Voss und Lorenz Engell. Paderborn: Wilhelm Fink, S. 153-182.

Schwarz, Markus / Schwarz, Markus (2002). »Neue Techno-Platte. Sven Väth will sich entbehrlich machen. "In: Frankfurter Allgemeine Zeitung vom 27. März, http://www.faz.net/-gqz-3708 (Stand vom 29.1.2017).

Settle, Mark. »Memebusting. Even without turntables, you're still a DJ.« In: DJWORX vom 19. Mai 2015, http://djworx.com/memebusting-even-withoutturntables-youre-still-a-dj (Stand vom 29.1.2017).

Sonnenfeld, Alexander (2011). Bewegungslehre. o.0.: Eigenpublikation.

Spielmann, Yvonne (2002). »Medienästhetik. Voraussetzungen und Grundlagen.« In: Einführung in die Medienwissenschaft. Konzeptionen, Theorien, Methoden, Anwendungen. Hg. v. Gebhard Rusch. Opladen: Westdeutscher Verlag, S. 243-256.

St John, Graham (Hg.) (2004). Rave Culture and Religion. London u.a.: Routledge.

St John, Graham (2012). Global Tribe: Technology, Spirituality and Psytrance. Bristol: Equinox.

Turner, Dave (2016). »Carl Cox Will Spin Vinyl for the First Time in 10 Years for Last Residency Party. «In: Mixmag vom 14. September, http://mixmag.net/ $\mathrm{read} /$ carl-cox-will-spin-vinyl-for-the-first-time-in-10-years-at-the-music-is-revol ution-closing-news (Stand vom 29.1.2017).

uultraman (2012). [Online-Kommentar zu Deadmau5 (2012)]. In: Gizmodo vom 25. Juni, http://gizmodo.com/5921053/all-djs-are-glorified-button-pushers?comme $\mathrm{nt}=50924491 \#$ comments (Stand vom 29.1.2017).

Veen, Tobias C. van / Attias, Bernardo Alexander (2011). "Off the Record: Turntablism and Controllerism in the 21st Century (Part 1). «In: Dancecult. Journal of Electronic Dance Music Culture 3, Nr. 1, https://dj.dancecult.net/index. $\mathrm{php} /$ dancecult/article/view/319/313 (Stand vom 16.2.2017).

Vigh, Henrik (2008). „Crisis and Chronicity. Anthropological Perspecitves on Continuous Conflict and Decline. «In: Ethnos 73, Nr. 1, S. 5-24.

Vigh, Henrik (2009). »Motion Squared: A Second Look at the Concept of Social Navigation. «In: Anthropological Theory 9, Nr. 4, S. 419-438.

Wicke, Peter (2004). »Virtuosität als Ritual. Vom Guitar Hero zum DJ-Schamanen.« In: Musikalische Virtuosität. Hg. v. Heinz von Loesch, Ulrich Mahlert und Peter Rummenhöller. Mainz u.a.: Schott, S. 232-243.

\begin{abstract}
This article explores whether DJ skills and practices can be included in the concept of virtuosity. At first, difficulty as a basic requirement for virtuosity is analysed for different types of DJ performances. I then explain one of the DJ's principal skills, mixing: By focusing on beatmatching I evaluate its relevance for DJing in the digital age, ask if risk is a necessary ingredient in virtuosity and frame these arguments with practice-theoretical observations. In reference to socio-anthropological concepts I highlight the dramaturgical, performative, and social aspects of DJing and position the DJ as a »social navigator «. In so doing, I propose expanding the notion of virtuosity to include the specificities of DJ culture and electronic (dance) music, thereby updating the concept to be applied to contemporary popular music.
\end{abstract}

